\title{
AVALIAÇÃO DA APLICABILIDADE DA VERSÃO PORTUGUESA DE UM QUESTIONÁRIO DE COMPETÊNCIAS PARA ENFERMEIROS HOSPITALARES
}

\author{
Silvia Helena Henriques ${ }^{1}$, Mirelle Inácio Soares², Laura Andrian Leal ${ }^{3}$
}

\footnotetext{
${ }^{1}$ Doutora em Enfermagem. Professora do Departamento de Enfermagem Geral e Especializada da Escola de Enfermagem de Ribeirão Preto (EERP), da Universidade de São Paulo (USP). Ribeirão Preto, São Paulo, Brasil. E-mail: shcamelo@eerp.usp.br

${ }^{2}$ Doutoranda do Programa Interunidades de doutoramento em enfermagem da EERP/USP. Ribeirão Preto, São Paulo, Brasil. E-mail: mirelle_soares83@usp.br

${ }^{3}$ Mestre em Enfermagem Fundamental. Programa de Pós-Graduação Enfermagem Fundamental da EERP/USP. Ribeirão Preto, São Paulo, Brasil. E-mail: laura.andrian.leal@usp.br
}

\section{RESUMO}

Objetivo: realizar avaliações psicométricas da versão adaptada do Competence Evaluation Questionnaire para enfermeiros brasileiros no contexto hospitalar, avaliando a confiabilidade da estrutura interna desta versão.

Método: trata-se de um estudo metodológico, no qual a amostra constituiu-se de 273 enfermeiros pertencentes a duas instituições hospitalares públicas. O instrumento é composto por 27 itens distribuídos em cinco domínios de competências. Para validade de constructo da versão adaptada do instrumento, verificou-se Alpha de Cronbach, Análise Fatorial Confirmatória e Exploratória e calculado novamente o Alpha de Cronbach dos domínios do instrumento, a fim de validar seu novo arranjo estrutural.

Resultados: foi verificado Alpha de Cronbach total de 0,923 e por domínios variando de 0,397 (Gerenciamento) a 0,833 (Processo de enfermagem). Na análise fatorial foram encontrados valores de Índice de Ajuste Comparativo e Índice de Tucker-Lewis, respectivamente, 0,808 e 0,783, demonstrando valores aceitáveis e próximos do ideal. Na análise fatorial exploratória, o índice de Kaiser-Meyer-Okin foi de 0,918 e o teste de esfericidade de Bartlett teve valor $\mathrm{p}<0,00$, ocorrendo uma nova disposição estrutural do instrumento que posteriormente se pode confirmar a consistência interna dos novos domínios variando de 0,732 a 0,845 e, portanto, todos os valores consistentes.

Conclusão: a versão para o português brasileiro do Questionário de Avaliação de Competências ficou composta por 27 itens agrupados aos domínios a que pertencem obedecendo aos critérios da Análise Fatorial exploratória realizada, revelando-se instrumento adequado, válido e confiável.

DESCRITORES: Enfermeiros. Hospitais. Competência profissional. Tradução. Escalas.

\section{APPLICABILITY ASSESSMENT OF THE PORTUGUESE VERSION OF A COMPETENCY QUESTIONNAIRE FOR HOSPITAL NURSES}

\footnotetext{
ABSTRACT

Objective: to develop psychometric assessments of the adapted version of the Competency Evaluation Questionnaire for Brazilian nurses in the hospital context, assessing the reliability of the internal structure of this version.

Method: a methodological study was undertaken, in which the sample consisted of 273 nurses from two public hospitals. The scale consists of 27 items, distributed in five competence domains. For the construct validity of the adapted version of the instrument, Cronbach's Alpha was verified, Confirmatory and Exploratory Factor Analysis was applied and Cronbach's Alpha was again calculated for the questionnaire domains, aiming to validate its new structural arrangement.

Results: the total Cronbach's Alpha coefficient was 0.923, while the coefficients per domain ranged from 0.397 (Management) to 0.833 (Nursing process. In the factor analysis, a Comparative Fit Index and Tucker-Lewis Index of 0.808 and 0.783 were found, respectively, demonstrating acceptable and near-ideal values. In the exploratory factor analysis, the Kaiser-Meyer-Olkin index was 0.918 and the result of Bartlett's sphericity test was $\mathrm{p}<0.00$. After developing a new structural arrangement of the scale, the internal consistency of the new domains was confirmed and ranged from 0.732 to 0.845 , that is, all indices were consistent.

Conclusion: the Brazilian Portuguese version of the Competence Evaluation Questionnaire consisted of 27 items, grouped in the domains they belong to. The criteria of the exploratory factor analysis applied were met, showing an appropriate, valid and reliability instrument. DESCRIPTORS: Nurses. Hospitals. Professional competence. Translating. Scales.
} 


\title{
EVALUACIÓN DE LA APLICABILIDAD DE LA VERSIÓN PORTUGUESA DE UN CUESTIONARIO DE COMPETENCIAS PARA LOS ENFERMEROS HOSPITALARIOS
}

\begin{abstract}
RESUMEN
Objetivo: realizar evaluaciones psicométricas de la versión adaptada del Competence Evaluation Questionnaire para los enfermeros brasileños en el contexto hospitalario y evaluando la confiabilidad de la estructura interna de esta versión.

Método: se trata de un estudio metodológico y constituido por una muestra de 273 enfermeros pertenecientes a dos (2) instituciones hospitalarias públicas. El instrumento está compuesto por 27 ítems distribuidos en cinco dominios de competencias. Para la validez del constructo de la versión adaptada del instrumento se verificó el Alpha de Cronbach, el Análisis Factorial Confirmatorio y Exploratorio y se calculó nuevamente el Alpha de Cronbach de los dominios del instrumento, a fin de validar su nuevo orden estructural.

Resultados: se verificó el Alpha de Cronbach total de 0,923 y por dominios variando de 0,397 (Gerenciamiento) a 0,833 (Proceso de enfermería). En el análisis factorial se encontraron los valores del Índice de Ajuste Comparativo e Índice de Tucker-Lewis, respectivamente, de 0,808 y 0,783 , demostrando valores aceptables y próximos de lo ideal. En el análisis factorial exploratorio, el índice de Kaiser-Meyer-Okin fue de 0,918 y el test de esfericidad de Bartlett tuvo un valor de $p<0,00$, ocurriendo una nueva disposición estructural del instrumento que posteriormente se pudo confirmar con la consistencia interna de los nuevos dominios variando de 0,732 a $0,845 \mathrm{y}$, por lo tanto, todos los valores consistentes.

Conclusión: la versión para el portugués brasileño del Cuestionario de Evaluación de Competencias quedó compuesta por 27 ítems agrupados con los dominios al que pertenecen y obedeciendo a los criterios del Análisis Factorial exploratorio realizado, rebelándose como un instrumento adecuado, válido y confiable.
\end{abstract}

DESCRIPORES: Enfermeros. Hospitales. Competencia profesional. Traducción. Escalas.

\section{INTRODUÇÃO}

No âmbito hospitalar, a temática competência profissional tem se constituído foco de atenção dos administradores hospitalares, bem como dos enfermeiros. No seu processo de trabalho, os enfermeiros assumem os cuidados aos pacientes mais graves, desenvolvendo de forma compartilhada atividades assistenciais e gerenciais, ${ }^{1}$ necessitando de competências específicas a fim de garantir a excelência da qualidade da assistência.

Nesse contexto, as competências representam um conjunto de conhecimentos, habilidades e atitudes que cada trabalhador possui e que serve de referencial para construção de seu desenvolvimento na empresa. ${ }^{2}$ Desse modo, as competências profissionais envolvem esses três eixos, sendo os conhecimentos compreendidos como o saber do profissional; as habilidades, entendidas como o saber fazer; e as atitudes definidas como saber agir, julgar, escolher e decidir em uma situação específica. ${ }^{3}$

Ao gerenciar pessoas, as empresas e/ou gestores devem proporcionar condições para que as competências individuais possam ser aproveitadas pela organização. Diante disso, a avaliação de desempenho por competências é uma estratégia da gestão de pessoas que pode auxiliar no diagnóstico do potencial dos trabalhadores e melhorar o seu desempenho. Para tal, importa não só para a adoção dos conhecimentos técnico-profissionais, como também na capacidade de mobilizar os indivíduos e a organização para enfrentar problemas no cotidiano de trabalho. ${ }^{2}$
A literatura internacional revela que pesquisadores têm se preocupado com essa questão e desenvolvido instrumentos para avaliar as competências de enfermeiros por meio de seu desempenho, tanto da área de saúde pública ${ }^{4}$ como da área hospitalar. ${ }^{5-6}$ No Brasil, pesquisadores têm identificado as competências de enfermeiros no hospital, ${ }^{7-8}$ utilizando-se de abordagens qualitativas. No entanto, até o momento da coleta de dados deste estudo, não foram encontrados estudos publicados que tivessem utilizado ferramentas quantitativas, como escalas validadas, para avaliar as competências de enfermeiros hospitalares.

Nessa perspectiva, o Competency Evaluation Questionnaire (CEQ) é uma ferramenta construída para avaliar as competências de enfermeiros, o qual utilizou declarações de instrumentos de avaliação de hospitais de ensino, públicos e privados. É uma escala do tipo Likert de cinco pontos que vai desde $1=$ baixa competência a $5=$ alta competência. Os itens do CEQ revelam padrões universais de cuidados da American Nurses' Association (ANA), Nursing and Midwifery Council (NMC) e do International Council of Nurses (ICN), e apresentam enunciados de competências distribuídos em cinco domínios, dentre eles: gerenciamento, profissionalismo, resolução de problemas, processo de enfermagem e conhecimento dos princípios básicos de enfermagem. ${ }^{5}$

Assim, à luz da extensa revisão da literatura e discussões de profissionais na área de recursos humanos em enfermagem, percebeu-se que as competências do CEQ relacionadas aos enfermeiros eram pertinentes ao contexto cultural brasileiro. Para 
seu uso, faz-se necessária a sua tradução e adaptação transcultural. Além disso, em continuidade às etapas de validação, torna-se necessário realizar avaliações psicométricas para a confirmação de sua confiabilidade para o uso no Brasil.

Diante dessa premissa, este estudo teve como objetivo realizar avaliações psicométricas da versão adaptada do CEQ para enfermeiros brasileiros no contexto hospitalar avaliando a confiabilidade da estrutura interna desta versão.

\section{MÉTODO}

Trata-se de um estudo do tipo metodológico que teve como foco verificar as propriedades psicométricas da versão portuguesa de uma escala de avaliação de competências.

O processo de adaptação transcultural do CEQ teve início com a autorização dos autores responsáveis pelo instrumento original. Assim, os referidos autores permitiram realizar todo o processo de adaptação transcultural do instrumento com a aplicação de testes psicométricos posteriores. Desse modo, uma abordagem muito empregada e utilizada neste estudo apresenta a seguinte sequência: tradução, retrotradução, revisão técnica da versão traduzida por comitê de juízes e piloto. ${ }^{9}$ Complementarmente às etapas de validação do instrumento, foram realizadas análises estatísticas para avaliar em que medida o instrumento pode, de fato, ser considerado válido para o contexto ao qual foi adaptado. Assim, adaptar e validar um instrumento são, portanto, passos distintos, porém complementares. ${ }^{10}$

Ressalta-se que a primeira etapa se iniciou com a tradução do instrumento original em inglês para a língua portuguesa por dois tradutores bilíngues. As duas versões em português em conjunto com a versão original em inglês foram avaliadas por um Comitê de especialistas (docentes de enfermagem, enfermeiros hospitalares e dois linguistas) com o objetivo de compará-las e elencar os itens que mantiveram a equivalência semântica com os itens do instrumento original, resultando na versão consensual português1. A terceira etapa constituiu-se da retrotradução da versão consensual português1 para o idioma original do instrumento; etapa esta realizada por outros dois tradutores bilíngues fluentes em inglês, de forma independente, às cegas, para a versão original do instrumento. A quarta etapa constituiu-se das revisões técnicas do instrumento com a finalidade de comparar essa versão traduzida às retrotraduções e o original em inglês, resultando na versão consensual português.
Essa versão foi submetida à avaliação de um comitê de cinco juízes formado por enfermeiros hospitalares que avaliaram a clareza, pertinência e aparência dos itens do instrumento. Acrescentados os ajustes sugeridos no instrumento, transcorreu-se para a etapa piloto, da qual participaram 29 enfermeiros hospitalares. No entanto, o título adotado para a versão adaptada do CEQ foi Questionário de Avaliação de Competências (QAC).

Em continuidade às etapas anteriormente citadas, foi realizada uma avaliação psicométrica do QAC a fim de verificar sua confiabilidade. Para tal, utilizou-se uma amostra não aleatória constituída por todos os profissionais elegíveis, presentes ou escalados, para o trabalho nos hospitais participantes durante o período de coleta de dados.

O cálculo da amostra para a avaliação das propriedades psicométricas do QAC partiu do pressuposto de que, para um instrumento contendo 27 questões, seriam necessários no mínimo dez profissionais para cada item a fim de se realizar a análise fatorial. ${ }^{11}$ Dessa forma, os critérios de inclusão da amostra foram: enfermeiros que trabalhavam nos referidos hospitais que tinham contato diretamente com os pacientes internados; enfermeiros que não tinham contato direto com o paciente, mas cujas funções desempenhadas no trabalho afetam diretamente o cuidado ao paciente internado (líderes, gerentes, supervisores); e estar presente em um dos turnos de trabalho no período de realização da coleta de dados na organização, sendo que a mesma ocorreu entre março e outubro de 2016.

Assim sendo, para a validade de constructo da versão adaptada do instrumento, verificou-se a consistência interna dos itens por meio do alfa de Cronbach. Após, foi realizada Análise Fatorial Confirmatória (AFC), Exploratória (AFE) e novamente AFC. ${ }^{12} \mathrm{Na}$ AFC, o ajustamento do modelo proposto foi avaliado pelos índices CFI (índice de ajuste comparativo) e TLI (índice de Tucker-Lewis), para os quais valores acima de 0,80 são aceitáveis, sendo considerados ótimos os valores acima de 0,95;13 e o Root Mean Square Error of Approximation (RMSEA), para o qual são esperados valores inferiores a $0,08 .{ }^{14}$ Posteriormente, a estrutura fatorial do instrumento foi avaliada por meio de Análise Fatorial Exploratória (AFE). Para verificar a adequação da análise fatorial, foi utilizado o índice de Kaiser-Meyer-Okin Measure of Sampling Adequacy $(\mathrm{KMO})$ e o teste de Esfericidade de Bartlett $(\mathrm{p}<0,05)$ que avalia a presença de correlações entre as variáveis.

Em continuidade, realizou-se o método de extração com Análise de Componentes Principais e Rotação Varimax Ortogonal. Como critério de sele- 
ção dos fatores, adotou-se autovalor maior que 1 e cargas significativas, aquelas superiores a $0,3 \cdot{ }^{15} \mathrm{Em}$ seguida, repetiu-se a análise fatorial confirmatória (AFC) com o intuito de validar a estrutura fatorial obtida na AFE. Após, calculou-se novamente o Alfa de Cronbach dos domínios do instrumento com o intuito de validar seu novo arranjo estrutural. Em todas as análises foi adotado o nível de significância de $5 \%(a l f a=0,05)$.

Esta pesquisa foi aprovada pelo Comitê de Ética em Pesquisa da Escola de Enfermagem de Ribeirão Preto da Universidade deSão Paulo, sendo registrada sob CAAE: 38544414.9.0000.5393 com o Parecer N. 243/2014, e os participantes assinaram o Termo de Consentimento Livre e Esclarecido (TCLE).

\section{RESULTADOS}

\section{O processo de adaptação transcultural}

Posteriormente à tradução e à adaptação, o QAC ficou composto por 27 itens, nos quais os respondentes deveriam se manifestar acrescentando valores de 1 a 5 , em que 1 = não se aplica a competência, 2 = baixa competência, $3=$ competência moderada, $4=$ boa competência e $5=$ excelente competência. A versão adaptada do instrumento para o português ficou composta por 27 itens distribuídos em cinco domínios de competências para enfermeiros, sendo eles: gerenciamento, profissionalismo, resolução de problemas, processo de enfermagem e conhecimento dos princípios básicos de enfermagem para enfermeiros hospitalares.

Em relação às características sociodemográficas dos participantes do piloto, $89 \%$ eram do sexo feminino, com idade variando de 26 a 44 anos, $45 \%$ com mais de 10 anos de experiência profissional e $48 \%$ entre cinco e 10 anos de atuação no hospital. Para o score total, o Alpha de Cronbach do piloto foi de 0,932, aproximando-se do instrumento original $(0,97)$, e variação entre 0,58 e 0,896 para os domínios, corroborando com um estudo recente que, ao validar um instrumento denominado Nurses Core Competence in Palliative Care, obteve um Alpha de Cronbach nos domínios do instrumento variando de 0,51 e $0,97 .{ }^{16}$

\section{Validade de constructo}

\section{Caracterização profissional e demográfica dos enfermeiros hospitalares}

Uma vez que o objetivo central deste estudo foi avaliar as propriedades psicométricas do QAC, foi aplicado em uma amostra de 273 enfermeiros atuantes em dois hospitais, dos quais $244(89,4 \%)$ eram do sexo feminino, sendo a média de idade entre eles de 38,06 anos. No que diz respeito a sua formação profissional, $90(33 \%)$ possuíam somente a Graduação, 128 (46,9\%) apresentavam algum tipo de Especialização, 46 (16\%) cursaram Mestrado e apenas nove (3\%) possuíam Doutorado, apresentando média de experiência profissional de 13,5 anos e média de tempo de serviço na instituição de 10,8 anos.

\section{Análise das respostas do QAC segundo os domínios}

Analisando as respostas dos enfermeiros participantes do estudo, observou-se uso constante das diversas competências elencadas no QAC. Assim, foi possível verificar que somente o item 12 Participação em pesquisas científicas e/ ou aplicação de resultados referente ao domínio Gerenciamento teve como maior ocorrência a competência moderada, com 33\% das respostas assinaladas; todos os demais itens de competências do QAC foram considerados pela maioria dos enfermeiros como boas ou excelentes, revelando que mesmo diante da realidade complexa do ambiente hospitalar, da constante atualização de tecnologias e de novas formas de relações de trabalho com a presença de equipes multiprofissionais, ocorre uma atuação comprometida dos enfermeiros, ou seja, ocorrem atitudes positivas com a busca de desenvolvimento de inúmeras competências profissionais.

\section{Validade e Confiabilidade do QAC}

Inicialmente, verificou-se a consistência interna dos itens, com Alpha de Cronbach total de 0,923, e por domínios apresentados 0,397 (Gerenciamento), 0,717 (Conhecimento dos princípios básicos), 0,746 (Profissionalismo), 0,808 (Resolução de Problemas) e 0,833 (Processo de enfermagem). Assim, observou-se Alpha de Cronbach consistente em quatro domínios do QAC.

Em continuidade, foi realizada $\mathrm{AFC}$, na qual foram encontrados valores de CFI e TLI, respectivamente, 0,808 e 0,783 , demonstrando valores aceitáveis para o índice CFI e abaixo para TLI; e o Root Mean Square Error of Approximation (RMSEA) foi 0,086 , o que não significa um bom ajuste do modelo proposto. No entanto, a fim de verificar a validade e fidelidade do instrumento, foi realizada AFE em que o índice de KMO foi de 0,918 e o teste de esfericidade de Bartlett teve valor de $x^{2}=3452,816$ com $p<0,001$. No teste de Comunalidades, evidenciou-se que todos os itens possuíam valores acima 
de 0,4 , variando em ordem decrescente dos valores de 0,714 a 0,406 .

Quanto à extração de fatores, a variância total explicada apresentou cinco autovalores superiores a 1,00 com uma porcentagem cumulativa de $58 \%$ do total da variância, sugerindo que o instrumento poderia ser divido em cinco domínios similares ao instrumento original, porém com um arranjo diferente. Para verificação desses domínios procedeu-se à rotação Varimax, apresentada na tabela 1.

Tabela 1 - Matriz das cargas fatoriais com Rotação Varimax do QAC segundo o novo arranjo estabelecido. Ribeirão Preto, SP, Brasil, 2017. (n=273)

\begin{tabular}{|c|c|c|c|c|c|}
\hline $\begin{array}{c}\text { Itens/Domínio } \\
\end{array}$ & 1 & 2 & 3 & 4 & 5 \\
\hline 27.Comunicação das atividades com a equipe de enfermagem & 0,721 & & & & \\
\hline 26.Documentação das atividades da enfermagem & 0,645 & & & & \\
\hline 6.Comunicação com colegas enfermeiros & 0,635 & & & & \\
\hline 3.Comunicação com pacientes e seus familiares & 0,629 & & & & \\
\hline 25.Manutenção da segurança do paciente & 0,594 & & & & \\
\hline 4.Comunicação com médicos e demais membros da equipe multidisciplinar & 0,578 & & & & \\
\hline 19.Manejo adequado de pacientes críticos & & 0,745 & & & \\
\hline $\begin{array}{l}\text { 15.Habilidade para avaliar as necessidades do paciente (biológicas, psicológicas, so- } \\
\text { ciais e espirituais) }\end{array}$ & & 0,680 & & & \\
\hline 17.Habilidade em estabelecer prioridades na assistência ao paciente & & 0,613 & & & \\
\hline $\begin{array}{l}\text { 18.Execução das responsabilidades de enfermagem baseadas na fundamentação } \\
\text { científica apropriada }\end{array}$ & & 0,501 & & & \\
\hline 20.Uso eficiente do tempo no trabalho & & 0,478 & & & \\
\hline 22.Habilidades administrativas e de prestação de contas & & & 0,695 & & \\
\hline 23.Entusiasmo, motivação na condução das atividades de enfermagem & & & 0,638 & & \\
\hline 24.Aplicação apropriada da filosofia e procedimentos hospitalares & & & 0,618 & & \\
\hline 21.Geração de novos conhecimentos relacionados ao desenvolvimento da profissão & & & 0,570 & & \\
\hline 12.Participação em pesquisas científicas e/ou aplicação de resultados & & & 0,543 & & \\
\hline $\begin{array}{l}\text { 5.Comunicação com o pessoal administrativo do hospital (setor de recursos huma- } \\
\text { nos e finanças) }\end{array}$ & & & 0,514 & & \\
\hline 14.Habilidade para realizar as etapas do processo de enfermagem & & & & 0,758 & \\
\hline 13.Conhecimento das etapas do processo de enfermagem & & & & 0,685 & \\
\hline 16. Capacidade em realizar diagnósticos de enfermagem precisos e acurados & & & & 0,655 & \\
\hline 11.Atualização do conhecimento na área da enfermagem & & & & 0,467 & \\
\hline 10.Segurança na implementação das habilidades de enfermagem & & & & 0,443 & \\
\hline 2.Comprometimento com a pontualidade e carga horária de trabalho & & & & & 0,752 \\
\hline 1.Aparência pessoal e postura profissional & & & & & 0,558 \\
\hline 8.Compromisso com as diretrizes éticas da profissão & & & & & 0,556 \\
\hline 7.Cumprir e fazer cumprir com as normas e regulamentos do hospital & & & & & 0,528 \\
\hline $\begin{array}{l}\text { 9. Conhecimento conceitual da enfermagem (familiaridade com as ciências básicas e } \\
\text { conceitos teóricos da enfermagem) }\end{array}$ & & & & & 0,515 \\
\hline
\end{tabular}

A tabela 1 revela cargas fatoriais acima de 0,4 , sendo que o item 14 apresentou a maior carga fatorial de 0,752 .

Posteriormente, realizou-se a AFC, o que indicou melhora dos índices CFI e TLI com 0,869 e 0,853, respectivamente. O RMSEA = 0,071 e SRMR (Standardized Root Mean Square Residual) $=0,066$ se aproximando dos valores adequados e confirmando a validade do instrumento. Por fim, foi realizado Alpha de Cronbach para a nova estrutura do instrumento, no qual se pode confirmar a consistência interna dos novos domínios variando de 0,732 a
0,845 e, portanto, todos os valores consistentes.

Dessa forma, considerando a nova disposição e agrupamento dos itens, após análise fatorial, os domínios foram renomeados. Para o domínio Gerenciamento, foram incluídos os itens 5, 12, 21, 22, 23 e 24; no domínio Comunicação, os itens 3, 4, 6, 25, 26 e 27; para o domínio Resolução de Problemas, os itens 15, 17, 18, 19 e 20; para o domínio Processo de Enfermagem, os itens 10, 11, 13, 14 e 16; e para o domínio Profissionalismo, os itens 1, 2, 7, 8 e 9. A versão brasileira do QAC validado com seus respectivos domínios está disposta no quadro 1. 
Quadro 1 - Versão Brasileira do QAC após validação de constructo. Ribeirão Preto, SP, Brasil, 2017.

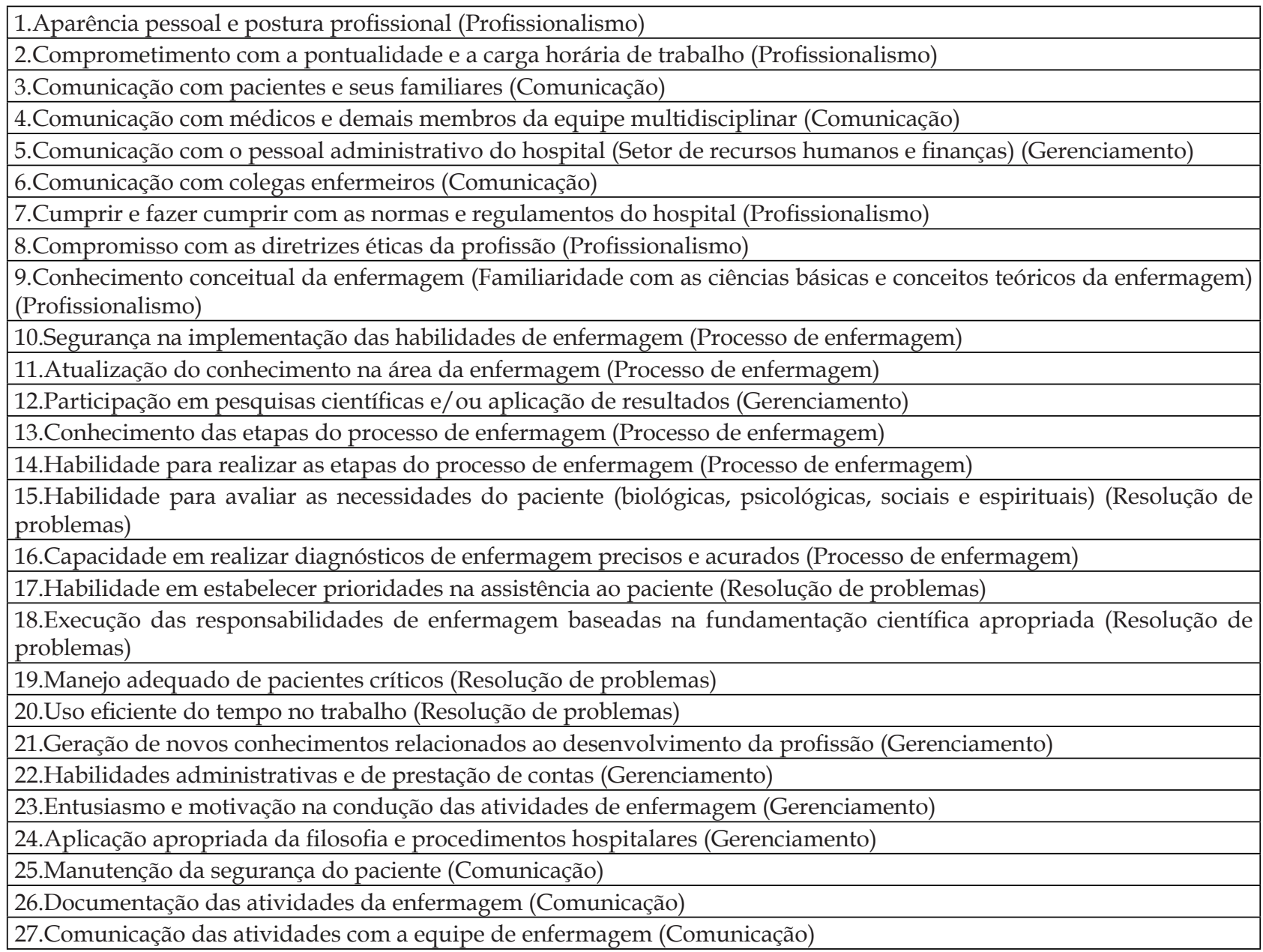

\section{DISCUSSÃO}

Com a crescente preocupação em garantir os cuidados prestados no contexto hospitalar e a necessidade de um quadro de enfermeiros com competências específicas, foram os fatores motivadores que levaram as pesquisadoras a buscarem um instrumento que pudesse avaliar o desempenho por meio das competências desses profissionais.

Nessa perspectiva, o CEQ foi uma ferramenta de âmbito internacional, construída para avaliar as competências de enfermeiros. Os autores da versão original do CEQ asseguraram a validação semântica, adaptação cultural no idioma inglês e piloto com 50 enfermeiros, no qual foi calculada a consistência interna dos itens apresentando Alpha de Cronbach $=0,97 \cdot{ }^{17}$ Posteriormente, foi testado em uma amostra de 258 egressos de enfermagem com formações diversas, provenientes de áreas rurais e urbanas do país de origem avaliando as suas competências para atuarem em hospitais comparando o sexo, a formação acadêmica e o tipo de hospital. Contudo, destaca-se que não foram realizados outros testes psicométricos referentes ao instrumento original nessa etapa. ${ }^{17}$

Ressalta-se que esta investigação, após o processo de adaptação transcultural, pretendeu avaliar a aplicabilidade da versão adaptada do CEQ. Nessa direção, é notório enfatizar que o processo de adaptação transcultural é uma etapa complexa que exige cautela, de modo que a versão obtida seja adequada ao constructo e equivalente à versão original. ${ }^{10}$

Dessa forma, visando atender a esses critérios, a tradução foi realizada por dois tradutores independentes juramentados, que são profissionais habilitados a emitir traduções públicas oficiais. No processo de adaptação do CEQ, a validade de conteúdo, incluindo equivalências semânticas, culturais, idiomáticas e conceituais, foi realizada por um Comitê de especialistas. Processo este es- 
sencial para obtenção de um instrumento com características psicométricas satisfatórias. ${ }^{18}$ Conforme descrito anteriormente, o CEQ é composto de 27 itens distribuídos em cinco domínios. No processo de adaptação do mesmo, foram realizados ajustes mínimos nas expressões dos itens 1, 4, 7, 9, 11, 13, $17,19,20,21,23$ e 27, resultando na versão adaptada denominada de Questionário de avaliação de competências (QAC).

Assim, reiterando as informações, para avaliar as propriedades psicométricas, foi utilizada uma amostra de 273 enfermeiros que atuavam em dois hospitais de grande porte. Considerando as respostas dos enfermeiros ao QAC, destacam-se os itens dois e oito, em que $69,2 \%$ e $72,2 \%$ dos profissionais identificaram como excelentes competências pertencentes aos domínios gerenciamento e profissionalismo do instrumento original, corroborando a pesquisa realizada pelos autores do CEQ na qual as maiores médias de respostas ficaram entre estes mesmos domínios. ${ }^{5}$ Essas respostas dizem respeito ao elevado comprometimento do enfermeiro com o seu local de trabalho, realização profissional nas atividades desenvolvidas dentro do contexto organizacional, compromisso com as diretrizes éticas da profissão, além de indicar que o estilo de gestão deve estar gerando motivação e satisfação, favorecendo o desempenho dessas competências pelos enfermeiros.

No entanto, para a avaliação das propriedades psicométricas do QAC, foram aferidas a sua consistência interna e a validade de constructo. A análise da consistência interna mensurada pelo Alpha de Cronbach foi de 0,923, sendo considerado excelente valor quanto mais próximos de 1 e para estudos conclusivos acima de 0,80 , sabendo-se que a análise da consistência interna como parâmetro para mensuração da confiabilidade de questionário constitui critério importante para a qualidade de estudos de adaptação. ${ }^{19-20}$

Para a validade de constructo do QAC, foi utilizada Análise Fatorial, sendo que a princípio, considerando-se uma estrutura teórica já existente, optou-se pela AFC a fim de verificar se o modelo se adequava ao modelo original. A AFC mostrou índice de CFI aceitável, TLI e RMSEA abaixo do valor adequado, o que significa que o modelo original necessita de ajuste. Assim, considerando que o TLI e o CFI são medidas que comparam o modelo proposto com a estrutura original, na tentativa de melhorar o ajustamento do modelo, procedeu-se à AFE. Nessa análise, o índice KMO próximo a 1 e o teste de esfericidades de Barlett com $p<0,05$ revelaram uma matriz adequada com correlação homogênea entre os itens do instrumento. ${ }^{21}$

Além da análise do KMO e do Teste de esfericidade de Barlett, empregou-se também a verificação das Comunalidades $\left(\mathrm{H}^{2}\right)$. A comunalidade indica a quantia total da variância que uma variável original compartilha com todas as outras variáveis incluídas na análise. ${ }^{19}$ Neste estudo, todos os itens apresentaram valores superiores a 0,4 , indicando que nenhuma variável deveria ser excluída. Simultaneamente, foi realizado o método de extração dos fatores, o que sugeriu a extração de cinco fatores com valores próprios superiores a 1, contribuindo, assim, para explicar a variância nas variáveis originais.

Em seguida, a rotação dos fatores foi efetuada e de modo a tornar o resultado empírico facilmente interpretável sem afetar as propriedades estatísticas, optando-se pela rotação ortogonal Varimax para minimizar o número de variáveis que apresentavam altas cargas em cada fator. ${ }^{20}$ Dessa forma, para interpretar o papel que cada variável tinha na definição de cada fator, foi assumido como valor mínimo aceitável uma carga fatorial $>0,45 .{ }^{15}$ Nesse sentido, a variância total explicada sinalizou que a escala poderia ser dividida em cinco domínios semelhantes ao instrumento original, porém com um novo arranjo entre os itens. A nova apresentação revelou que os itens são válidos por apresentarem carga fatorial maior do que 0,4 e, portanto, nenhum item deve ser excluído na adaptação da versão original do QAC.

Posteriormente, foi realizada a AFC em que se observou melhora nos índices de CFI, TLI e RMSEA, demonstrando que a nova estrutura é mais adequada para ser utilizada. Entretanto, houve um novo arranjo dos domínios com os itens, porém não houve retirada de qualquer item.

Nessa direção, cabe enfatizar que todos os itens apresentaram afinidades teóricas com seus respectivos domínios, sendo identificado novo Alpha de Cronbach para os domínios do instrumento confirmando adequado índice de confiabilidade em todos os domínios, sendo o instrumento considerado apropriado para mensurar as competências desenvolvidas pelos enfermeiros no âmbito hospitalar.

No entanto, considera-se uma limitação neste estudo pelo fato da amostra pertencer apenas a duas instituições hospitalares. Novas pesquisas fornecerão sustentabilidade para consolidar a validade do instrumento, além de corroborar para seu fortalecimento como instrumento de avaliação de desempenho dos profissionais nas organizações hospitalares. 


\section{CONCLUSÃO}

Instrumentos para avaliação de competências de enfermeiros hospitalares devem auxiliar futuros profissionais, gestores e pesquisadores a realizarem diagnósticos de competências de trabalhadores para sua determinada função a fim de realizar avaliações de desempenho de cada profissional, bem como favorecer a elaboração de treinamento e desenvolvimento de seu capital humano.

Este artigo apresentou a avaliação psicométrica realizada da versão portuguesa adaptada denominada de QAC, o qual tem o objetivo de avaliar as competências dos enfermeiros que atuam em hospitais. Observa-se que os resultados psicométricos encontrados na amostra utilizada se apresentaram adequados. Assim, a estrutura final do QAC ficou composta por 27 itens que foram agrupados aos domínios a que pertencem obedecendo aos critérios da AFE realizada.

Percebe-se que o novo arranjo dos itens com os domínios ficou com uma representação mais favorável que o modelo original, ressaltando que mantiveram o número de cinco domínios de competências, entretanto houve substituição de um termo visando melhor compreensão, ficando assim denominados: Processo de enfermagem, Gerenciamento, Comunicação, Resolução de problemas e Profissionalismo.

Assim, espera-se que futuramente o QAC possa ser adotado em investigações científicas no intuito de estabelecer dados normativos das competências de enfermeiros hospitalares, avaliando a sensibilidade deste instrumento antes e após intervenções, como também poderá auxiliar os gestores de serviços de enfermagem na avaliação de seus colaboradores.

\section{REFERÊNCIAS}

1. Camelo SHH. Professional competences of nurse to work in Intensive Care Units: an integrative review. Rev Latino-am Enfermagem [Internet]. 2012 [cited 2017 Mar 28]; 20(1):192-200. Available from: http:/ / goo.gl/m3KCQ0

2. Oliveira DA, Gonçalves RS, Barbosa ACQ. Percepção dos gestores de recursos humanos em relação ao modelo de gestão por competências. Rev FSA [Internet]. 2014 [cited 2017 Mar 28]; 11(2):01-26. Available from:http:/ / www4.fsanet.com.br/revista/ index.php/fsa/article/viewFile/363/316

3. CameloSHH, Angerami ELS. Professional competence: the building of concepts and strategies developed by health services and implications for nursing. Texto Contexto Enferm [Internet]. 2013 [cited 2017 Apr 10];
22(2):552-60. Available from: http:/ / www.scielo.br/ pdf/tce/v22n2/v22n2a34.pdf

4. Kalb KB, Cherry NM, Kauzloric J, Brender A, Green $\mathrm{K}$, Miyagawa L, et al. A competency-based approach to public health nursing performance appraisal. Public Health Nurs [Internet]. 2006 [cited 2017 Mar 28]; 23(2):115-38. Available from: http:/ / www.ncbi. nlm.nih.gov/pubmed/16684187

5. Safadi R, Jaradeh M, Bandak A, Froelicher E. Competence assessment of nursing graduates of Jordanian universities. Nurs Health Sci [Internet]. 2010 [cited 2017 Mar 28]; 12(2):147-54. Available from: http:/ / www.ncbi.nlm.nih.gov/pubmed/20602685

6. Nilsson J, Johansson E, Egmar AC, Florin J, Leksell J, Lepp $\mathrm{M}$, et al. Development and validation of a new tool measuring nurses self-reported professional competence- The Nurse Professional Competence (NPC) Scale. Nurse Educ Today [Internet]. 2014 [cited 2017 Mar 28]; 34(4):574-80. Available from http:/ / www.ncbi.nlm.nih.gov/pubmed/23938092

7. Camelo SHH, Rocha FLR, Chaves LDP, Silva VLS, Soares MI. Competencias profesionales y estrategias organizacionales de gerentes de enfermería. Cienc Enferm [Internet]. 2016 [cited 2017 Mar 28]; 22(1):7586. Available from: http:/ / www.scielo.cl/pdf/cienf/ v22n1/art_07.pdf

8. Paiva KCM, Junior WJS. Competências profissionais de enfermeiros e sua gestão em um hospital particular. Rev Bras Enferm [Internet]. 2012 [cited 2017 Mar 28]; 65(6):899-90. Available from: http:/ / www.scielo.br/scielo.php?script=sci_arttext\&pid =S0034-71672012000600003

9. Guillemin F, Bombardier C, Beaton DE. Cross-cultural adaptation of health related quality of life measures: literature review and proposed guidelines. J Clin Epidemiol. 1993; 46(12):1417-32.

10. Borsa JC, Damásio BF, Bandeira D. Adaptação e validação de instrumentos psicológicos entre culturas: algumas considerações. Paidéia [Internet]. 2012 [cited 2017 Mar 28]; 22(53):423-32. Available from: http:/ / www.scielo.br/pdf/paideia/v22n53/14.pdf

11. Crocker L, Algina J. Introduction to Classical and Modern Test Theory. New York (US): CBS College Publishing; 1986.

12. Yuan KH, Bentler PM. Structural equation modeling. In: C Rao, S Sinharay. 'Handbook of Statistics: Psychometrics'. Handbook of Statistics, Elsevier; 2007. chapter 10.

13. Brown TA. Confirmatory Factor Analysis for Applied Research. Second edition. New York: The Guilford Press; 2015.

14. Pilati R, Laros JA. Modelos de equações estruturais em psicologia: conceitos e aplicações. Psic: Teor e Pesq [Internet]. 2007 [cited 2017 Mar 28]; 23(2):20516. Available from: http://www.scielo.br/scielo. php? pid=S0102-37722007000200011\&script $=$ sci_ abstract\&tlng $=\mathrm{pt}$ 
15. Hair Junior JF, Black WC, Babin BJ, Anderson RE, Tatham RL. Multivariate Data analysis. Seventh Edition. Upper Saddle River: Prentice Hall; 2010.

16. Slatten K, Hatlevik O, Fagerstrom L. Validation of a new instrument for self-assessment of nurses core competencies in palliative care. Nurs Res Pract [Internet]. 2014 [cited 2017 Mar 28]:1-8. Available from: http:/ / www.hindawi.com/journals/ nrp/2014/615498/

17. Sperber AD. Translation and validation of study instruments for cross-cultural research. Gastroenterel [Internet]. 2004 [cited 2017 Mar 28]; 126(1 Suppl. 1):124-8. Available from: http://www.ncbi.nlm.nih. gov/pubmed/14978648

18. Beaton DE, Bombardier C, Guillemin F, Ferraz MB.
Guidelines for the process of cross-cultural adaptation of self report measures. Spine. 2000; 25(24):3186-91.

19. Vieira VA. Escalas em Marketing: métricas de resposta do consumidor e de desempenho empresarial. São Paulo: Editora Atlas S.A.; 2011.

20. Kerwee CB, Screllingerhout JM, Vergagen AP, Koes BW, De Vet HC. Methodological quality of studies on the measurement properties of neck pain and disability questionnaires: a systematic review. J Manipulative Physiol Ther [Internet]. 2011 [cited 2017 Mar 28]; 34(4):261-72. Available from: https:/ / www. ncbi.nlm.nih.gov/pubmed/21621728

21. Pallant J. SPSS survival manual: A step by step guide to data analysis using SPSS. 6th ed. McGraw-Hill Education; 2016.
Correspondência: Silvia Helena Henriques Camelo. Universidade de São Paulo

Escola de Enfermagem de Ribeirão Preto,

Departamento de Enfermagem Geral e Especializada

Avenida dos Bandeirantes, 3900 - Campus Universitário

14040-902 - Monte Alegre, Ribeirão Preto, SP, Brasil

E-mail: shcamelo@eerp.usp.br
Recebido: 10 de abril de 2017

Aprovado: 08 de novembro de 2017

This is an Open Access article distributed under the terms of the Creative Commons (CC BY). 